\title{
ФИНАНСОВО-ЭКОНОМИЧЕСКИЕ АСПЕКТЫ СТРАТЕГИЧЕСКОГО РАЗВИТИЯ ПРЕДПРИНИМАТЕЛЬСКОЙ ДЕЯТЕЛЬНОСТИ
}

\author{
(C) 2021 Михель Е.А. \\ Санкт-Петербургский политехнический университет Петра Великого, Россия, Санкт-Петербург \\ E-mail:mihel_ea@spbstu.ru
}

Сложившаяся практика осуществления стратегического развития предпринимательской деятельности во многом определяется финансовыми и экономическими возможностями предприятий. В современных условиях многократно возрастают требования к максимизации финансовоэкономической эффективности от ведения бизнеса, поскольку поддержание стандартного (находящегося в статическом состоянии) уровня предпринимательской эффективности неприемлемо в динамично изменяющемся макроэкономическом пространстве. Сложившиеся обстоятельства подтверждают актуальность проведения исследований по анализу финансово-экономических компонентных составляющих в бизнес-процессах компаний. Настоящая статья посвящена проработке вопросов стратегического развития предпринимательской деятельности с акцентом на финансово-экономические элементы хозяйствования, динамика которых способна обеспечить повышение конкурентоспособности и создать устойчивый потенциал для поступающего развития предприятий. Для этого предлагается проанализировать теоретические аспекты концептуальной значимости финансовых и экономических элементов в построении стратегического развития предприятия. В результате появляется возможность отобрать набор показателей для оценки стратегических возможностей предпринимательской деятельности, учитывая потенциальное взаимодействие предприятия с контрагентами (конкурентами и партнерами). Предложенный подход планируется в дальнейшем апробировать на машиностроительных предприятиях для совершенствования их стратегического развития, что позволит проработать инструментарий взаимодействия предприятий с учетом их положения в отрасли и финансовых возможностей.

Ключевые слова: стратегическое развитие, финансово-экономические аспекты, стратегия развития, предпринимательская деятельность, взаимодействие предприятий, стратегическое партнерство.

\section{Введение}

Предпринимательская деятельность играет существенную роль в экономическом развитии любого современного государства. В наиболее развитых странах достижение положительных социально-экономических эффектов напрямую зависит от механизмов реализации национальной политики воздействия на предпринимательский сектор и создания благоприятных условий для развития рыночной инфраструктуры [1]. Для функционирования предпринимательского сектора реализуемая политика должна учитывать макроэкономические факторы и определять предпочтительную стратегическую ориентацию предприятий по созданию устойчивой конкурентоспособности, производству продукции и услуг с заранее заданным уровнем качества [2; 3]. В итоге происходит модификация системы партнерских отношений и их эволюционная трансформация.
Потребность в расширении теоретических и методологических подходов к реализации предпринимательской деятельности актуализирует поиск путей повышения эффективности корпоративных бизнес-процессов. Специфические особенности стратегического планирования в данном контексте воспринимаются как составная часть системы управления, способная адаптироваться к конкретным ситуационным условиям ведения предпринимательской деятельности [4]. Стратегическое планирование следует рассматривать как реализацию структурированных аналитических процессов, логика которых направлена на определение будущего положения бизнес-субъекта в непрерывно изменяющемся макроэкономическом пространстве. Данный факт является необходимым условием стратегического развития предпринимательской деятельности. Однако трансформационные изменения определили значимость финансовых 
и экономических возможностей экономических субъектов, которые на текущий момент определяют стратегический потенциал бизнеса.

Актуальность проведения исследований в данном направлении подтверждается многократно возрастающими требованиями к максимизации финансово-экономической эффективности от ведения бизнеса, поскольку поддержание стандартного уровня предпринимательской эффективности является категорически неприемлемым в динамично изменяющемся экономическом пространстве. Требуется учесть сложившиеся обстоятельства для анализа финансово-экономических компонентных составляющих в бизнес-процессах компаний. В итоге появляются возможности заложить в стратегических траекториях развития комбинации внутренних и внешних условий осуществления хозяйствования, рациональное использование эффектов от которых позволяет сократить или полностью нивелировать негативное воздействие внутренних и внешних экстерналий.

Цель исследования заключается в проведении проработки вопросов стратегического развития предпринимательской деятельности с акцентом на финансово-экономические элементы хозяйствования.

\section{Материалы и методы исследования}

При написании статьи были рассмотрены труды исследователей по различным экономическим направлениям, в первую очередь связанных со стратегическим анализом и планированием, а также финансовым анализом и экономикой предприятия. Исследование строится на использовании общенаучных методов (анализ, синтез и теоретическое обобщение), а также включает в себя другие специфические методы, позволяющие построить теоретическую базу и развить практические направления решения поставленной проблемы.

Результаты исследования и их обсуждение

В первой части настоящей статьи предлагается провести теоретический анализ стратегического развития предпринимательской деятельности, учитывая повышенную значимость финансово-экономических аспектов функционирования экономических субъектов и необходимости акцентировать на них повышенное научно-практическое внимание.

Рациональные и совершенно новые подходы к стратегическому управлению хозяй- ственной деятельностью гарантируют создание богатого ресурса для создания новых и совершенствования ранее действующих корпоративных стратегий. Глобальные вопросы к обеспечению стратегического развития экономических субъектов расширяют различные аспекты хозяйственной деятельности, адаптируя системы управления к ускоренной динамике внешних изменений [1]. Масштабные трансформации наблюдаются в эпоху цифровизации социальноэкономических систем, затрагивающие функциональный аппарат экономических субъектов. Именно цифровые условия стали детерминирующими явлениями в изменении предприятий, отраслей, регионов на основе внедрения сквозных цифровых технологии. Такие процессы затронули и промышленность, обуславливая пути детализированного развития предпринимательских стратегий [5].

Во многом сущностные характеристики и содержание аппарата стратегического управления связаны с экономической категорией финансов. Функциональная значимость использования финансовых ресурсов в предпринимательской деятельности приводит к необходимости расширения идентификации финансовых стратегий в составе общей стратегии развития предприятия [6]. Деловая практика на протяжении долгого времени расширяет функциональную значимость финансовых ресурсов с позиции формирования ни основе их рационального использования конкурентных сил, определяющих долгосрочную прибыльность бизнеса в любой отрасли. Так, формулирование финансовой стратегии позволяет расширять пути взаимодействия предприятия с его партнерами и конкурентами на рынке, создавая устойчивый конкурентный потенциал [7].

Финансовый элемент в комбинации с экономическими возможностями компании способствует ускорению корпоративного роста, в том числе за счет своевременного обновления фондов предприятия. В научной литературе имеется множество подходов к определению предпринимательской деятельности, однако все они учитывают необходимость управления финансовым и экономическим состоянием компании, учитывая ключевые характеристики предпринимательства: инициативность, целенаправленность, управляемость, рискованность, инновационность и доходность. Соответствием этим характеристикам обуславливает наличие 
способностей для непрерывного совершенствования инструментов поддержания устойчивости предпринимательской деятельности, особенно в условиях глобальной нестабильности, которые наблюдаются сегодня на рынке [3; 5].

В рамках исследования предлагается сконцентрировать внимание на вопросах взаимодействия между отдельными экономическими субъектами, заложив конкретные элементы данных функциональных взаимосвязей в состав стратегии корпоративного развития. Базовым элементом в данном направлении является стратегическое планирование предпринимательской деятельности, которое осуществляется на основе определения внутрифирменных планов, а также путем планирования корпоративной детальности на внешнем рынке. Таким образом, в рамках стратегического планирования требуется обосновать фундаментальные положения для формирования порядковой системы распределения ресурсов между сферами деятельности предприятия с учетом уже сложившегося или потенциального взаимодействия с другими экономическими субъектами, катализируя базис финансово-экономических аспектов. При этом характеристики представленного взаимодействия в системе стратегического планирования должны учитывать целый ряд аспектов предпринимательской деятельности, например, отраслевые особенности, материально-финансовые возможности бизнес-субъекта, территорию расположения предприятия и функционирования производств, различного рода дифференциации и так далее [4].

Стратегическое планирование на предприятии можно представить в виде следующей последовательной системы:

формирование долгосрочных стратегических установок

$\rightarrow$ разработка внутреннего функционала компании и создание соответствующих структурных подразделений

$\rightarrow$ разработка целей по всем структурным подразделениям

$\rightarrow$ мониторинг функционирования оперативной деятельности подразделений и проведение ситуационного анализа

$\rightarrow$ координация тактических действий и своевременное внесение плановых и/или внеплановых изменений $\rightarrow$ осуществление комплексного контроля за достижением плановых установок

$\rightarrow$ корректировка стратегии и формирование новых долгосрочных планов. Можно сделать выводы, что стратегическое планирование строится на основе выявления целевых установок предприятия, сильных и слабых сторон его развития, потенциальных рисках недостижения плановых показателей и так далее. Особое значение следует уделить финансово-экономическим аспектам, отражение которых в стратегии развития позволит сформировывать расширенный комплекс прогнозов и предложений по корректировке системы планирования.

Сложность стратегического планирования заключается в многофакторной экстраполяции, которая зачастую не позволяет строить высококачественные модели повышения эффективности предпринимательской деятельности без сокращения угроз и поиска новых возможностей, к которым следует отнести и грамотную стратегию взаимодействия между различными бизнес-субъектами [8; 9]. Эффективность такого взаимодействия с контрагентами предприятия во многом определяется не только состоянием конкуренции, но и потенциалом выстроенных партнерских или конкурентных связей. Для этого требуется своевременно анализировать общность интересов и схожесть целевых установок, проводя непрерывный анализ информационных потоков. Принципиально важно при взаимодействии с контрагентами-партнерами обеспечить доверие, взаимовыгодные условия и долгосрочную перспективность.

С авторской позиции построение межсубъектных взаимосвязей с высоким качеством макроустойчивости позволяет определить перспективные траектории стратегического планирования, однако для любой стратегии требуется принимать наиболее рациональные управленческие решения, минимизируя вероятность наступления негативных последствий. Для этого требуется разрабатывать наборы показателей и факторных условий для каждой конкретной ситуации, которая определяется миссией и целями, заложенными в общей стратегии развития. Принятие такого рода решений и отбор наиболее значимых наборов показателей осуществляется в первую очередь на основе аналитических и статистических данных, выделении в них параметрических зависимостей и провалов, учет которых позволяет модернизировать долгосрочное функционирование компании, своевременно исправляя проблемы во взаимодействии с контрагентами и выявляя новые возможности 
для сотрудничества [3].

Следовательно, стратегическое планирование является ключевым элементом стратегического развития. Данный элемент определяет стратегические цели и пути их достижения. Для этого разрабатываются и обосновываются методы и модели стратегического анализа, их качественная интеграция. Концептуальная значимость программного подхода в области стратегического управления расширяется в связи с необходимостью интегрировать предпринимательские конкурентные стратегии, закладывая в них финансово-экономические аспекты хозяйственного функционирования [9]. Важной составляющей программы стратегического развития является экономически правильный выбор направлений применения инструментов планирования и достижение на основе их использования нового уровня технико-экономических и эксплуатационных показателей предприятия [2], а также более эффективного использования ресурсов [10].

Стратегическое планирование является значимым элементом в достижении целей и задач предприятия, а использование экономикоматематического инструментария позволяет определять ключевые формы и способы ведения деятельности, выстраивать грамотные подходы для достижения заданных в стратегии параметров [11; 12]. К такому инструментарию относятся и критерии теории игр. Для реализации теоретико-игрового инструментария к вопросам стратегического развития предпринимательской деятельности требуется выделить перечень определенных показателей, использование и трансформация которых напрямую влияет на итоговые результаты предпринимательской деятельности и достижения заложенных в ней целевых установок стратегии. Использование теоретико-игровых подходов позволяет с высоким уровнем эффективности смоделировать потенциальное поведение различных экономических агентов в процессе ведения конкурентной борьбы или осуществления определенных мероприятий, направленных на сотрудничество. Так, финансовая стратегия является важным элементом корпоративного управления, в ее состав входят решения по многим внутрифирменным вопросам, а теоретико-игровые критерии позволяют рационализировать управленческую деятельность и взаимодействие между субъектами по многим финансовым вопросам. На основе полученных данных определяется успешность деловой активности и эффективность распределяемых денежных потоков [13; 14]. В исследовании предлагается за расчетные параметры принять финансово-экономические аспекты экономической деятельности предприятий для определения их стратегических перспектив при активной стадии взаимодействия.

Рыночные условия не позволяют добиться успеха в бизнесе без аккумулирования и использования информационных потоков, способствующих формированию объективных оценок о состоянии предприятия, целевых рынках и основных конкурентах. Экономикоматематический аппарат позволяет использовать совокупность методов и подходов для совершенствования бизнес-процессов через поиск наилучших способов достижения стратегических целей. Поддержание устойчивого развития является весомо значимым направлением, что в последнее время особенно актуализируется в связи с усиленным влиянием глобальных экстерналий, к которым можно отнести и пандемию COVID-19. Предприятия ищут способы стратегического поддержания устойчивого развития на всех уровнях управления. Так, многократно повышается значимость инструментальных подходов, позволяющих учитывать неполноту информации [15; 16].

Проведение оценки действующих стратегий предпринимательских структур и выделение на основе полученных данных наиболее эффективных подходов к построению стратегий предприятий и взаимодействия с контрагентами определяют целесообразность рассмотрения финансово-экономических аспектов предпринимательской деятельности, их динамику и структурное изменение в зависимости от конкретных условий взаимодействия с различными агентами. Непосредственное выделение качественных зависимостей позволит разработать алгоритмы стратегического развития, учитывая потребность в непрерывном совершенствовании функционала предприятия, опираясь на принятие управленческих решений с максимальной вероятностью получения новой эффективности. Стратегическое планирование требует от управленческого персонала проявлять способность к аналитической деятельности, что позволяет оценить степень конкуренции между предприятиями на основе анализа их финансово-экономического состояния, положе- 
ния субъектов в отрасли, а также на основе возможностей по взаимодействию во взаимовыгодных сегментах.

Стратегический выбор участников взаимодействия предоставляет полезную для процесса принятия управленческих решений информацию. Качество прогнозов определяет наличие ключевых факторов успеха стратегий предпринимателей, а также потенциал их трансформации. При проведении конкурентного анализа учет отраслевой специфики связан с определением доминирующих финансовоэкономических показателей и идентификацией их потенциальных изменений. Данный факт позволяет выявить альтернативные пути стратегического развития компании и учесть данные альтернативы в стратегическом плане [6; 17; 18]. Альтернативные модели, выстроенные на основе прогнозов способствуют разработке более совершенных механизмов взаимодействия в системе стратегического управления предпринимательской деятельностью. Таким образом, невозможно поддерживать стратегическую эффективность, находясь в статическом состоянии. Требуется осознавать качественную динамику рынка, информационные потоки и изменчивость в поведении экономических агентов, поскольку лишь в таком случае возможно создать устойчивый потенциал для поступающего развития предпринимательской деятельности [4].

Место стратегического планирования в системе стратегического развития предприятия схематически рассмотрено на рисунке 1 . Осуществление эффективного планирования связано с учетом потоков данных по самому предприятию и со стороны его внешнего окружения. Для проведения комплексных расчетов в экономико-математических моделях требу- ется отбирать наиболее весомые показатели, осуществляя выборку и анализ располагаемых данных с учетом их статического или динамического состояния. Отобранные значения и рассчитанные показатели должны быть заложены в системе стратегического планирования предпринимательской деятельности. В составе стратегического развития происходит разработка комплексных подходов с учетом всех заложенных альтернатив.

Системный подход к стратегическому развитию должен учитывать условия рыночной трансформации окружающего экономического пространства, формируя инструменты воздействия для своевременного выявления и противодействия непрерывно возрастающему риску. Таким образом, при разработке аппарата стратегического развития требуется формировать эффективные модели мониторинга и оценки финансово-экономического риска [19,20]. На базе полученных знаний о природе предпринимательской деятельности возможно выявить качественные факторы, определяющие предпосылки ее формирования и последствия от наступления тех или иных обстоятельств, что требуется учитывать при распределении финансовых ресурсов и достижении экономических целей. Практическая реализация построенной стратегии обуславливает наличие организационноуправленческих факторов, позволяющих учитывать в выработанных направлениях отдельные структурные подразделения предприятия, их функционирование между собой, а также внешнее влияние на хозяйственную систему предприятия. С этим эффективно справляется внутрикорпоративная организационная система, позволяющая выстраивать надстройки алгоритмов практического внедрения стратегии. С по-

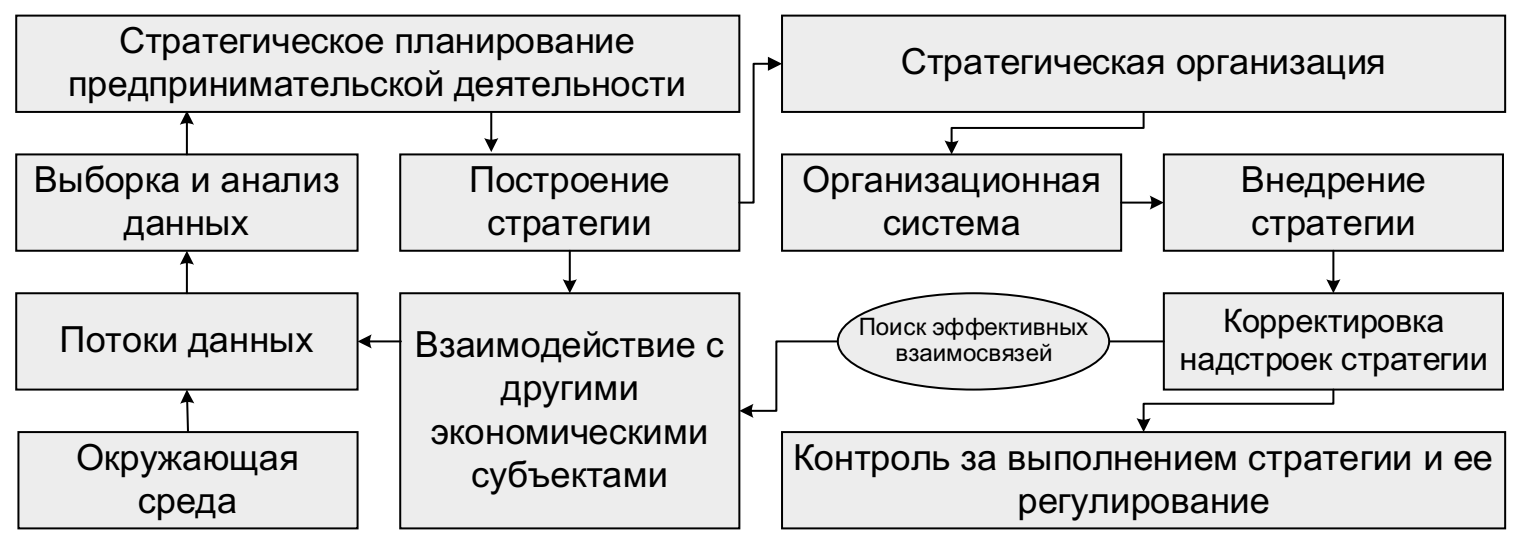

Puc. 1. Стратегическое планирование в системе стратегического развития 
зиции взаимодействия предприятия с экономическими агентами в разрабатываемой стратегии должны быть заложены аспекты конкуренции и сотрудничества, которые трансформируются с корректировками надстроек стратегии для поиска более эффективных и устойчивых взаимосвязей. Контроль за исполнением стратегии, ее регулированием и своевременными корректировками позволяет проводить мониторинг достижения ключевых показателей, формируя новые направления в стратегическом планировании предпринимательской деятельности [4; 21].

Непременным условием обеспечения стратегических возможностей финансового развития предприятия является признание ключевого положения финансовых аспектов системе корпоративного управления. Финансовоэкономический аспект в стратегии компании гарантирует создание эффективности в системе стратегического управления, так как затрагивает области обеспечения финансовыми ресурсами, в частности привлечение капитала в другие функциональные стратегии, а также оказывает прямое воздействие на финансовую и рыночную устойчивость организации в долгосрочной перспективе [17]. Успех деловой активности зависит от положительных результатов хозяйственной деятельности, которых невозможно достичь без учета финансово-экономических аспектов в стратегии. Модель стратегического предпринимательства строится на эффективном использовании вводимых в хозяйственные процессы ресурсов [18]. Поддержание финансовой независимости предприятия создает устойчивый стратегический потенциал для противостояния реальным и потенциальным рискам и угрозам.

С методологической точки зрения, учет финансово-экономического аспекта в стратегии позволит повысить качественную отдачу от таких элементов, как планирование, прогнозирование и моделирование. Таким образом, у предприятия появляется возможность реализовывать более мощные инструменты реализации стратегических целей в динамично изменяющихся условиях. Во второй части исследования предлагается акцентировать внимание на финансово-экономических условиях стратегического развития предпринимательской деятельности в сфере машиностроения.

К объективным причинам возрастающей значимости изучения стратегического развития машиностроительного производства можно отнести: сложность процесса управления промышленным предприятием в связи со структурной разветвленностью; необходимость установления и документального закрепления ключевых источников предпринимательского успеха как внутри, так и за пределами реализации предпринимательской деятельности; непрерывно усложняющиеся требования к постановке представления о конкурентных преимуществах предприятия; развитие способов применения сценарного и ситуационного подходов в целях стратегического планирования предпринимательской деятельности и так далее. Следовательно, рациональный процесс проектирования желаемого будущего промышленного предприятия обуславливает поиск эффективных путей его достижения не только через производственные составляющие, но и за счет учета факторов окружающей среды.

При анализе особенностей ведения предпринимательской деятельности в сфере машиностроения выделяется ряд проблем, которые препятствуют достижению стратегических целей и задач: нарушение баланса влияния между линейными руководителями и плановыми отделами (структурными единицами); несовершенство механизма практической реализации долгосрочных решений в условиях сложной динамики производства; недостаточный уровень профессиональных знаний, опыта и компетенций в управленческой среде по вопросам стратегического планирования и т.д. Значимость финансово-экономических аспектов стратегического развития предпринимательской деятельности позволяет взаимоувязать финансовоэкономические показатели предприятия с системой управления рисками и оценке потенциальных возможностей предприятия, что позволит предотвратить потенциальную потерю конкурентоспособности бизнес-субъекта в условиях рыночных отношений за счет внедрения гибкой системы своевременной реакции на внешние изменения [8; 22].

Рассмотрение действующих стратегий предпринимателей в сфере машиностроения предлагается осуществлять на основе модернизации классической модели стратегического управления Р. Линча [1], отдавая предпочтение именно ситуационному подходу, задав в системе параметры, учитывающие влияние финансово- 
экономических аспектов на стратегическое развитие предприятия. Схематически базис данной системы представлен на рисунке 2.

В заложенном направлении стратегическое планирование выступает значимым составным элементом стратегического развития предприятия и решает наиболее значимые для предпринимательской деятельности задачи: определение комплексной стратегии развития; выявление и обоснование параметров конкурентной стратегии; проработка элементов функциональных стратегий. Учет финансово-экономических аспектов через критериальный отбор ключевых показателей при оценке стратегий предпринимателей позволит выделить факторы формирования альтернатив стратегического развития, определить роль стратегического планирования в достижении ключевых целей и задач, разработать возможности по расширению или сужению взаимодействия с другими экономическими субъектами (в том числе и взаимодействие с тремя и большим количеством субъектов) [23].

Совершенствование бизнес-процессов требует от стратегического планирования предпринимательской деятельности производить учет параметров финансово-экономического положения предприятия, определяя тенденции его ретроспективного развития для построения достоверных прогнозов. Полученные аналитические данные и экономико-математические модели позволяют выработать практические методы и подходы к улучшению функционирования системы хозяйствования, что предоставит возможность руководству принимать более эффективные организационно-управленческие решения в условиях высокого уровня неопределенности. Проведение количественных расче- тов отобранного набора показателей позволит задать траектории оптимальных положений корпоративной стратегии. Наиболее обобщенно алгоритм стратегического планирования можно представить в виде смены обобщенных этапов: $\mathrm{Sp}=\mathrm{Gs} \rightarrow \mathrm{Sa} \rightarrow \mathrm{Sf} \rightarrow \mathrm{Js} \rightarrow \mathrm{Dbp}$, где: Gs - целеполагание; Sa - стратегический анализ; Sf - стратегический прогноз; Js - обоснование стратегии; Dbp - разработка бизнес-проектов. Предлагаемый алгоритм обосновывается действующими теоретическими и методологическими подходами к исследованию элементов стратегического развития в функционировании предпринимательской деятельности [3]. Для построения экономико-математических моделей и выделения критериальных зависимостей требуется проводить количественные измерения отдельных параметров, а финансово-экономические показатели могут стать наиболее очевидным условием подбора критериев для выработки инструментального аппарата стратегического планирования и комплексного развития предпринимательской деятельности.

Требуется отметить, что весомо значимым составным элементом стратегического развития производства является экономически правильный выбор направлений применения доступных инструментов, способных учесть наибольший комплекс требований к достижению нового уровня технико-экономических и эксплуатационных показателей производственного предприятия. Существенным аспектом производственно-хозяйственной деятельности промышленных предприятий является разработка плана создания новых мощностей на заранее определяемый уровень основных технико-экономических и эксплуатационных

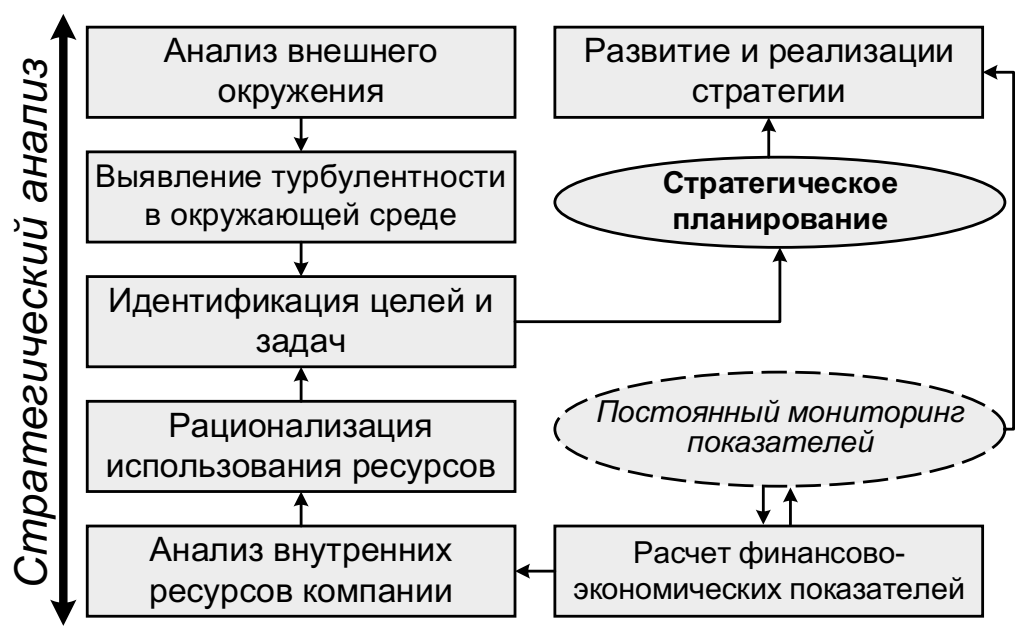

Puc. 2. Параметры финансово-экономических аспектов в системе стратегического развития 
показателях производственного предприятия [2]. Для этого целесообразно анализировать ключевые параметры финансово-экономического развития предприятия [22]. В частности, теоретико-игровые критерии могут стать базисом перераспределения разновероятностных событий для построения альтернатив действия менеджеров и использования инструментов, соответствующих конкретной ситуации. Необходимо отметить, что данная практика также позволит развить условия для внедрения новых инициатив в масштабах всего предприятия, благоприятно влияя на производственную продуктивность и результативность его деятельности [15].

Поскольку для определения траекторий развития машиностроительных предприятий необходимо задать видение их стратегических позиций, то допустимо рассмотреть в ретроспективе их финансово-экономическое положение в различные периоды и исследовать факторы внутренней и внешней среды, влияющие на изменение целевых установок предпринимательской деятельности, например, конкурентное положение предприятия, его капитализации и доходная часть. Особенности стратегического развития предпринимательской деятельности в машиностроении связаны также с такими факторами, как высокая социально-экономическая значимость производимой продукции и большая конкуренция на рынке, что требуется учитывать при построении экономических механизмов [3; 24].

Принимая во внимание, что в рыночных условиях предприятия функционируют как сетевой элемент взаимодействия, где каждое деловое решение или действие одного игрока влияет на других бизнес-субъектов, которые находятся на рынке, взаимодействуют и конкурируют с различными предприятиями. Для развития системы взаимодействия в контексте стратегического развития предпринимательской деятельности в сфере машиностроения предлагается рассмотреть финансово-экономические показатели. На основе полученных данных допустимо в дальнейшем произвести авторскую разработку экономико-математического инструментария по комплексному развитию стратегического планирования предпринимательской деятельности, основываясь на теоретико-игровых подходах.

Для достижения поставленной цели предлагается рассмотреть следующие финансовоэкономические элементы: структура имущества предприятия и источники его формирования; деловая активность; финансовые результаты. Перечисленные показатели можно использовать для определения стратегических перспектив предприятия и выявления возможностей для сотрудничества между собой или с третьими сторонами. Схематически взаимодействие предприятия с другими экономическими субъектами и влияния данных представлены на рисунке 3.

\section{Заключение}

Оптимальность стратегического развития предпринимательской деятельности заключается в достижении экономической эффективности бизнеса, которую можно максимизировать путем получения сетевых эффектов для нескольких игроков от грамотно выстроенного межсубъектного взаимодействия. В первую очередь это обуславливается получением устойчивых преимуществ за счет технологических и организационных взаимосвязей. В ма-

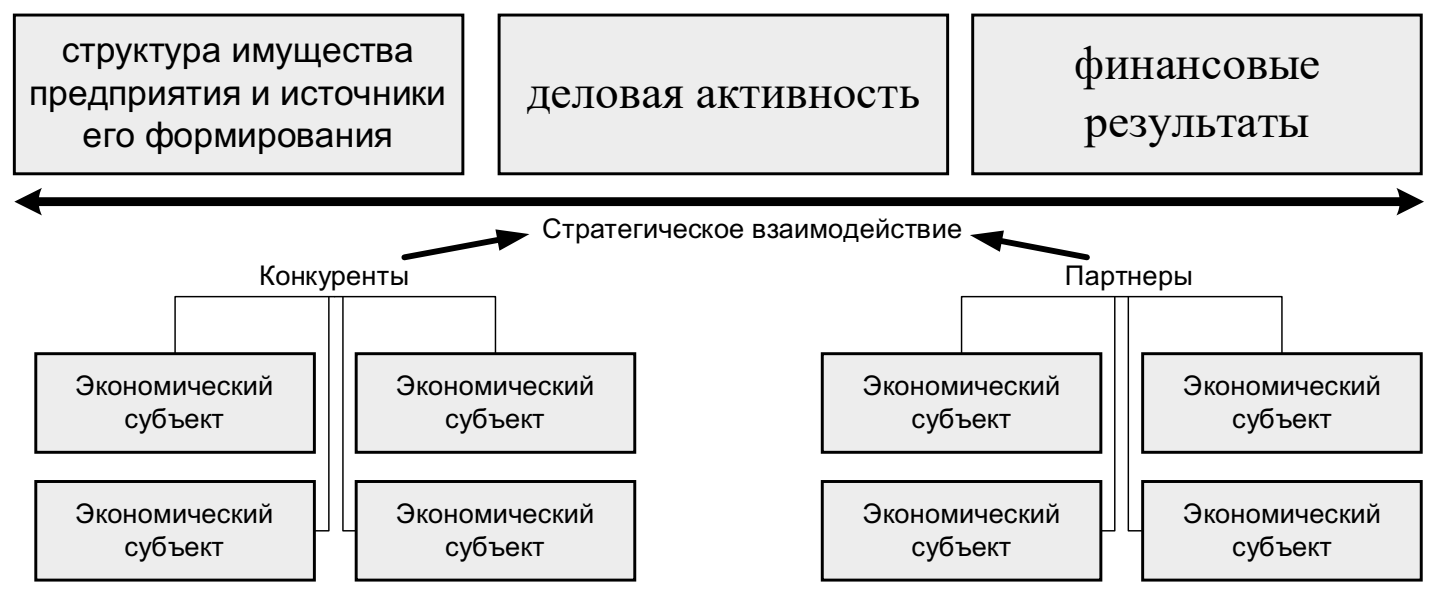

Puc. 3. Схематическое влияние бизнес-субъектов на финансово-экономические показатели предприятия 
шиностроительном комплексе принципиальную значимость для предпринимательской деятельности приобретают условия грамотно выстроенной системы взаимодействия, которая способствует формированию кумулятивных производственно-хозяйственных эффектов в стратегическом планировании, в первую очередь путем рационального распределения финансовых ресурсов для получения новых экономических эффектов. Проведение расширенной оптимизации в современных экономических условиях является базисом достижения стабильных конкурентных преимуществ на базе совершенствования аппарата межкорпоративного взаимодействия. Требуется производить комплексный факторный анализ внутренней и внешней среды, выстраивать систему принятия наиболее рациональных управленческих решений.

В долгосрочной перспективе обеспечение качественного неструктурного роста конкурен- тоспособности машиностроительных предприятий является качественным фактором концептуальной проработки вопросов стратегического планирования путем построения оптимизационных моделей. Стратегическое развитие предпринимательской деятельности связано с созданием условия достижения сопоставимых эффектов с минимальными затратами. Для этого целесообразно производить учет финансовоэкономических аспектов, что в итоге позволит отобрать набор показателей для оценки стратегических возможностей предпринимательской деятельности, учитывая потенциальное взаимодействие предприятия с его контрагентами (конкурентами и партнерами). Авторский подход может существенно развить практику совершенствования стратегического развития машиностроительных предприятий за счет проработки инструментария межкорпоративного взаимодействия с учетом положения каждого отдельного субъекта в отрасли.

\section{Библиографический список}

1. Linch R. Corporate Strategy. 4-th. ed. Pitman Publishing, 2007. 864 p.

2. Айрапетова А.Г., Корелин В.В., Мепория Г.Г. Стратегическое планирование как метод развития производственного потенциала предприятия // Известия СПбГЭУ. 2021. № 3. С. 121-125.

3. Стратегическое развитие малого бизнеса и формы поддержки индивидуального предпринимательства. Н. Новгород: Профессиональная наука, 2018. 302 с.

4. Филобокова Л.Ю., Дробкова О.С. Стратегическое управление развитием мегарегиона и его подсистемой «малое предпринимательство» на основе модели интеграционно-сбалансированного взаимодействия (на материалах Московской области). Тамбов: Юком, 2017. 161 с. DOI: 10.17117/mon.2017.09.03

5. Цифровизация экономических систем: теория и практика / под ред. А.В.Бабкина. СПб: Политех-Пресс, 2020. 796 c. DOI: $10.18720 /$ IEP/2020.3

6. Родионов Д.Г., Рудская И.А. Стратегическое управление финансами предприятия // Российский экономический интернет-журнал. 2018. № 4. С. 95.

7. Porter M. E. The Five Competitive Forces That Shape Strategy // Harvard business Review. 2008. № 86. pp. $78-93$.

8. Хуссейн Л.А. Роль стратегического планирования в развитии производительности предприятия // Вестник УГНТУ. Наука, образование, экономика. Серия: Экономика. 2018. № 1. С. 98-103.

9. Dhliwayo S. Entrepreneurship and Competitive Strategy: An Integrative Approach // Journal of Entrepreneurship. 2014. No 1.pp. 115-135. DOI: 10.1177/0971355713513356

10. Zaytsev A., Kozlov M., Kichigin O. Rental analysis of innovation component in resource productivity // В сборнике: IOP Conference Series: Materials Science and Engineering. 2019. С. 012064.

11. Лисогор Г.И.Экономико-математическое моделирование - основа сценарного стратегического планирования // Вестник УГНТУ. Наука, образование, экономика. Серия: Экономика. 2016. № 2. С. 37-41.

12. Стрельцова Е.Д., Бородин А.И., Яковенко И.В. Модельный инструментарий стратегического управления промышленным предприятием // Прикладная информатика. 2018. № 4. С. 15-28.

13. Зайцев А.А., МихельЕ.А., Дмитриев Н.Д. Использование теоретико-игрового подхода для формирования финансовой стратегии взаимодействия предприятий // Бизнес. Образование. Право. 2020. № 3. С. 81-87.

14. Михель Е.А., Зайцев А.А., Родионов Д.Г.Построение теоретико-игровой модели взаимодействия промышленных предприятий // Бизнес. Образование. Право. 2020. № 4. C. 94-101. DOI: 10.25683/VOLBI.2020.52.359

15. Гайсина Г.Н., Курбангалиева А. Т. Совершенствование процесса бизнес-планирования на предприятии в современных условиях // Вестник научных конференций. 2015. № 3-3. С. 43-44. 
16. Родионов Д.Г., Дмитриев Н. Д., Дубаневич Л. Э. Построение эконометрической модели устойчивого развития промышленного предприятия // Вестник Алтайской академии экономики и права. 2021. № 7-1. С. 61-71. DOI: $10.17513 /$ vaael.1781

17. Хезазна Б. К вопросу о содержании финансовой стратегии корпорации // Финансы и управление. 2019 . № 3. C. 61-71. DOI: 10.25136/2409-7802.2019.3.30719

18. Awang A., Kassim A., NoorA.M., Shukor N., ShaariA.Z., Amran S., SelamatS.M., Khalid S.A.Strategic Entrepreneurship Model for Economic Transformation: Malaysian Evidence // Asian Social Science. 2015. No 7. pp. 19-34. DOI:10.5539/ass.v11n7p19

19. Зайцев А.А., Родионов Д.Г., ДубаневичЛ.Э., Ильченко С.В. Аудит и управление рисками при реализации инвестиционных проектов интеллектуальной направленности // Вестник Алтайской академии экономики и права. 2021. № 7-2. С. 152-162. DOI: 10.17513/vaael.1793

20. Зайщев А. А. Диагностика рентной устойчивости аграрного сектора экономики // Вестник Ленинградского государственного университета им. А. С. Пушкина. 2015. Т. 6. № 1. С. 5-18.

21. Bruton G.D., Filatotchev I., Si S., Wright M. Entrepreneurship and Strategy in Emerging Economies // Strategic Entrepreneurship Journal. 2013. No 3. pp. 169-180. DOI: 10.1002/sej.1159

22. Кузьменко О.В., Чернышов Д. С. Роль стратегического планирования в повышении устойчивости развития предприятия // Концепт. 2019. № 1. С. 121-125. DOI: 10.24411/2304-120X-2019-14009

23. Иввонен Л.А., Широкова Г.В. Стратегическое предпринимательство: сущность и основные направления исследований // Вестник Санкт-Петербургского университета. Менеджмент. 2016. № 4. С. 21-53.

24. Бардина Е. М. Особенности стратегического планирования предпринимательской деятельности в интегрированных структурах // Вестник ТГУ. 2012. № 3. С. 74-77. 